\title{
QoS based Performance Analysis of EAODV Protocol in Overlay Network
}

\author{
Hisham Alsaghier \\ College of Computer \& Information \\ Sciences \\ Majmaah University (KSA) \\ Saudi Arabia
}

\author{
S Mishra \\ College of Computer \& Information \\ Sciences \\ Majmaah University (KSA) \\ Saudi Arabia
}

\begin{abstract}
An overlay network comprises overlay nodes that are responsible for routing and forwarding, connected by overlay links that correspond to paths in the underlying network. The end-nodes in overlay networks are highly connected to each other due to flexible routing. This architecture have two major components; overlay nodes with virtual links, and the native layer over which the overlay network is built, and ensure performance and availability of internet routing, multicasting, QoS guarantees. This paper complements the current research on routing in Ad hoc network by proposing a new protocol EADOV. The performance of various routing protocol in mobile wireless network for UPD-based application are measured. Network Simulator NS2 on Fedora environment is used for simulation which included two mobile nodes with four types of traffic VoIP, video, CBR and FTP for creating heavy load and to simulate the protocols. QoS based performance metrics (PSNR, throughput, frame losses end-toend delay, bandwidth utilization and Error-Resilience for both sender and receiver) under different scenario has been done and results are compared for both existing and proposed routing algorithms. Better average PSNR, throughput, minimum end to end delay and less I frame (the main key frame in video which neither regenerated at destination) losses are achieved compare to AODV. Received video was also compared for both algorithms, the EAODV give better output and more good quality frames than AODV.
\end{abstract}

Keywords: AODV, EAODV, CDN, CBR, P2P, VoD. VoIP

\section{INTRODUCTION}

An overlay is a set of servers deployed beyond the internet that provide infrastructure to one or more applications, forwarding and handling data differently than internet and also it is coherent in nature [1]. Overlay networks have a network semantic layer above the basic transport protocol level that organizes the network topology according the nodes' together with bounded lookup times [3].Overlays are used to increase routing robustness, to increase security, to reduce duplicate messages and they do not need new equipment or modifications to existing software or protocols [4] [5]. Flooding-based systems do not scale well because of the bandwidth and processing requirement they place on the network, and they accommodate no guarantees as to lookup time or content accessibility. Overlay networks can address these concerns [2].In recent years, overlay networks have rapidly evolved and emerged as a promising platform to deploy new applications and services in the Internet. An example of an overlay network is the Gnutella [11] file sharing network, in which the overlay nodes are the end systems. These nodes correlate to each other to form an overlay network on which queries from nodes are forwarded by flooding. Other examples include research networks such as PlanetLab [15] and commercial networks such as Akamai [8]. The PlanetLab network consists of approximately four hundred nodes and allows users to configure the nodes into overlays for specific applications. In Akamai network the content distribution servers can also be considered an overlay network composed of approximately 15,000 nodes devoted to distributing content to end users from nodes nearest to them in the network topology. The SureRoute service [8] which runs on the Akamai network provides resiliency to websites by routing their traffic through an intermediate Akamai node when the direct path to the website is down.

The rest of the paper is organized as follows. Section II presents; Classification of Overlay Networks, Content Delivery Network (CDN), some typical parameters that would be useful to measure the performance analysis of $\mathrm{CDN}$ are presented in Section III. Reactive routing protocols with a comparison are listed chronologically in Section IV; Simulation results show that purposed algorithm EAODV give better average PSNR, throughput, less end-to-end delay and less I frame losses (the main key frame in video which is not regenerated at destination), presented in section $\mathrm{V}$. Concluding remarks followed by reference made in this paper in presenting the clarity of the philosophy of the research work.

\section{CLASSIFICATION OF OVERLAY NETWORKS}

Classification of overlay networks is a hard task as there are a large number of proposals and working overlay networks, exclusively designed for a specific objective with a different set of assumptions about its constituent nodes and links. Previously, the main classification of overlay networks has been based on the structure of the network. With the increase in overlays with dedicated nodes, those single axes of classification are insufficient to capture the variety of overlay networks that exist. To understand the design of overlay networks, it has been classified along three different axes, each grabbing a different aspect of a network's structure. The axes are based on the classification of nodes, the structure, management and design of the overlay networks. For each axis, there are some of the features and assumptions of the overlay networks that lie on that axis. Note that these axes are quite orthogonal, and so the same networks can appear in different classifications.

Overlays are characterized by the nodes that compose them. In the last century, most overlays were composed of nodes dedicated to the overlay. With the recent increases in connectivity to the Internet by end hosts, many overlays are composed only of end systems handled by users. These peerto-peer networks have seen wide acceptance and use. The first overlays were composed of nodes that were usually dedicated 
to the functioning of the overlay. More promptly, interest in such networks has increased for providing new and improved service on the Internet. These nodes tended to be connected to the overlay for long periods of time and had low failure rates. Thus, the algorithms for these overlay networks could mostly ignore the node behaviour. The nodes in these networks also tend to have better connectivity to the Internet [9], [10].Peerto-peer networks are composed of user-controlled end hosts. Therefore, their lifetimes on the peer-to-peer network vary and these networks have high churn in the nodes that constitute them. Examples of such networks incorporate Gnutella [11], Chord [12] and SplitStream [13]. To reduce the impact of the high churn, some peer-to-peer networks propose a two-level architecture in which a few dedicated nodes, which connect to the network for longer periods of time, are used to enhance the performance of the network, e.g., supernodes in KaZaa [14].The organization of overlay networks can vary widely. In small networks, it is possible to have the nodes keep track of all the nodes in the overlay. This approach quickly becomes impossible as the size of the network increases. For large networks (nodes numbering greater than a few hundred), two different strategies are used: unstructured and structured.

A Content Delivery Network (CDN) supports the delivery of any type of dynamic content, along with various forms of interactive media streaming. It maintains multiple points of presence with clusters of servers(surrogate server).In CDN approach, client-server communication approach is replaced by two communication flow first between the client and the clusters of servers (surrogate server), and another between the clusters of servers and the origin server it reduces congestion and increases content distribution and availability. There are two approaches widely used, first is overlay model such as Akamai, Digital Island and Speedera [8, 16, 17]. Another approach is a network model, It arranges code to routers and switches so that they can recognize specific application types and make forwarding decisions on the basis of predefined policies .Generally, CDNs use the overlay model.

\section{CONTENT DELIVERY NETWORK (CDN)}

Content Delivery Networks (CDNs) give services that improve network performance by maximising information measure; Content Delivery Network consists of the many mobile nodes that act as each host furthermore as router within the free house air. Therefore Content Delivery Network works with none pre-existing infrastructure $[2,3,4$, 7]. The standard of service is huge challenge within the field of wireless Content Delivery Networks, since the mobile nodes square measure plug-n-play devices they need to face several problems in several applications of wireless communications.

The sector of wireless networking emerges from the mixing of non-public computing, cellular technology, and also the web. This is often as a result of the increasing interactions between communication and computing, that square measure dynamical info access from "anytime anywhere" into "all the time, everywhere." at this time, an outsized form of networks exist, starting from the well-known infrastructure of cellular networks to non-infrastructure wireless adhoc networks. Once any link of a multi-hop communication path breaks, the trail should be repaired or reconstructed. Throughout this process, packets could also be generated. This loss of packets can have an effect on quality of service (QoS) for each period of time and non-real-time applications and cause important turnout degradation [5,6]. Considering the recent advances in mobile content networking (e.g. WAP, IPv6 etc.), the infrastructure of mobile CDNs may play a leading role in terms of exploiting the emerging technological advances in the wireless web.

Video streaming over the Internet has become increasingly popular in recent years due to the rapid rise in network access speed of the end-users. Media streaming systems are distinct from file-sharing systems, in which a client has to download the entire file before using it. In a media streaming session, the receiver can already consume the tile while downloading. Video streaming applications are highly susceptible to packet delay and packet loss. A packet arriving after its scheduled playback time is useless and considered lost. A lost or corrupted frame creates an avalanche effect in the decoding process, as the decoding of subsequent frames is impaired by spatio-temporal error propagation. Therefore, in the case of loss, decoding is often completely stopped until the next Iframe arrives. In Internet video streaming, variations in transmission quality (throughput, delay etc.) are smoothed using a receiver buffer. The size of the buffer corresponds to the user-perceived initial delay of the application.

There are two approaches for Media streaming; client-server model and peer-to-peer approach, In client-server approach ,delay is minimum but cost is high in some cases like online games, resulting this server's bandwidth will be a bottleneck in the system due to the large number of client requests [65]. While in peer-to-peer approach, algorithms are designed so the clients have an active role in distributing the media content to other clients and thus remove the pressure from the server node[18] [19]. Advantages of media streaming are reported in [20]: like video search content monitoring, use of bandwidth, provides the content creator with more control over his intellectual property.

The term "streaming" used for video on demand on IP networks; usually referred to as "store and forward video", which was misleading nomenclature [21]. In wireless network there are various performance evaluation metrics are available. The most popular three metrics: throughput, PSNR, average PSNR and end-to end delay are been chosen to evaluate the performance of Content delivery network (CDNs) under different scenario.As the focus on performance improvement, In this paper performance of Content Delivery Network for various routing protocol are measured and compare them with EAODV Algorithm, also QoS based performance metrics (end-to-end delay, bandwidth utilization and Error-Resilience for both sender and receiver) using the enhanced AODV protocol in Content delivery network (CDNs) under different scenario has been done and results are compared.

\section{REACTIVE ROUTING PROTOCOLS}

There are differing kinds of routing protocols are offered, that's proactive, reactive and hybrid protocols. Reactive routing protocols for Content Delivery Networks are known as "on-demand" routing protocols like Ad hoc On Demand Distance Vector (AODV), Dynamic Source Routing (DSR) \& Temporarily Ordered Routing Algorithm (TORA) [22, 23, 24, 25]. In CDN, active routes could also be disconnected due to node quality. For route discovery Source Node broadcast Route-Request packet, each intermediate node gets a RouteRequest. This kind of protocols is usually based on flooding the network with Route Request (RREQ) and Route reply (RERP) messages. Other On Demand driven protocols are Associatively Based routing (ABR) [26], Signal Stability Based Adaptive Routing (SSA) [27],Location-Aided Routing Protocol (LAR) [28]. 
The Dynamic Source Routing (DSR) utilizes source routing algorithm. In this protocol, every data packet contains complete routing information to reach its dissemination. Furthermore, in DSR each node uses caching technology to maintain route information that it has learnt [24].

Source Routing (DSR, ABR and SSA) is good for smaller networks due to large data packet overhead .In AODV, no need to include the full path in the data packet and it Update Neighborhood information through periodic beacons .It is a Reactive Protocol, it discovers a route on demand and Nodes do not have to maintain routing information. Route Discovery is done by Hop by Hop basis i.e. If a source needs a route to a destination for which it does not already have a route in its cache, Source broadcasts Route Request (RREQ) message for specified destination, Intermediate node returns a route reply packet (RREP) (if route information about destination in its cache), or forwards the RREQ to its neighbors (if route information about destination not in its cache. If cannot respond to RREQ, increments hop count, saves info to implement a reverse path set up, to use when sending reply (assumes bidirectional link) i.e Source Node broadcast RouteRequest packet, Each intermediate node gets a RouteRequest and Establish a reverse link to node it received the RouteRequest from,If request received before it discard ,If route to destination is available and up-to-date itreturn RouteReply using the reverse link, Otherwise rebroadcast the RouteRequest ,Destination node respond with RouteReply using the reverse link. Route Maintenance in AODV is done in this way. When a node detects a link failure, it sends special RouteReply with infinity distance,RouteReply is propagated to source node and Source node initiates a new RouteRequest. Ad hoc On Demand Distance Vector (AODV) routing algorithmic rule could be a routing protocol designed for mobile ad hoc mobile networks or wireless network .In addition, AODV forms trees that connect multicast cluster members. The trees are composed of the cluster members and also the nodes required attaching the members. AODV uses sequence numbers to make sure that it is free from count to infinity problem also due to not included the full path in the data packet and it Update Neighborhood information through periodic beacons, establish time is less .It is a Reactive Protocol, it discovers a route on demand and Nodes do not have to maintain routing information [29]. Disadvantage of AODV is that the periodic beaconing leads to unnecessary bandwidth consumption. Proposed EAODV protocol overcomes the above shortcoming.

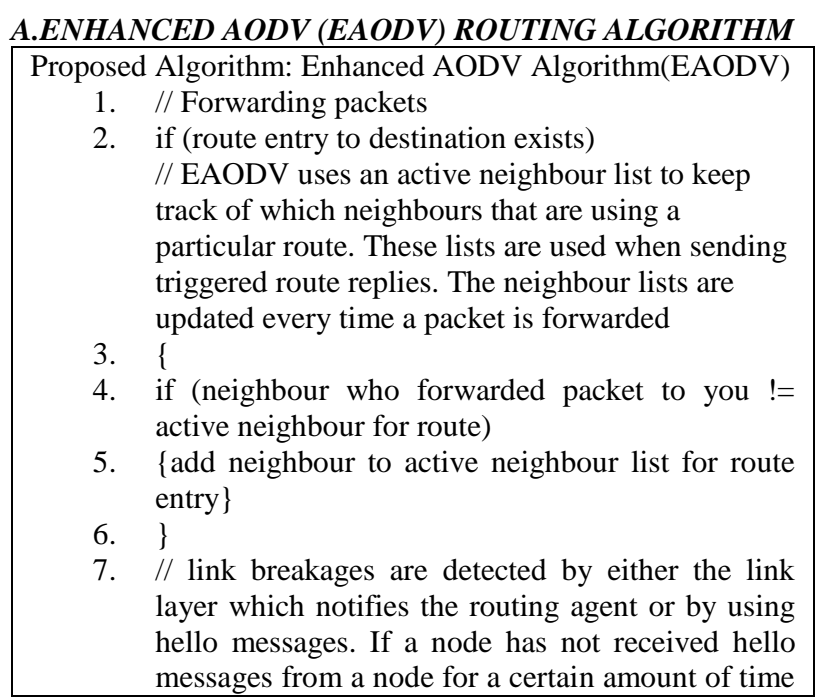

it will assume that the link is down. Every time a link is detected as down, EAODV will send a Triggered RREP to inform the affected sources(Sending Triggered RREP)

8. for (each address in the active neighbour list for a route entry)

9. \{create a link failure notice packet unicast to active neighbour

10. // every time a Triggered RREP is received informing about a broken link, the affected route entry must be deleted and neighbours using this entry must be informed.(Receiving Triggered RREP)

11. if (have active neighbours for broken route)

12. \{send Triggered RREP\}

13. delete route entry for broken route

In first block of algorithm lines 3-6, algorithm uses an active neighbor list to keep track of which neighbors' that are using a particular route. These lists are used when sending triggered route replies. The neighbor lists are updated every time a packet is forwarded.

In lines $8-9$, link breakages are detected by either the link layer which notifies the routing agent or by using hello messages. If a node has not received hello messages from a node for a certain amount of time it will assume that the link is down. Every time a link is detected as down, EAODV will send a Triggered RREP to inform the affected sources.

In lines 11-13, every time a Triggered RREP is received informing about a broken link, the affected route entry must be deleted and neighbors' using this entry must be informed.

\section{SIMULATION RESULT}

The performances of networks are evaluated in terms of different QoS parameter i.e. PSNR, throughput, frame losses end-to-end delay, bandwidth utilization and Error-Resilience for both sender and receiver.Framework for video transmission over the wireless network in NS2 on Fedora environment is used and simulation included two mobile nodes with four types of traffic VoIP, video, CBR and FTP for creating heavy load. The myEvalvid framework is used for adaptive cross-layer method for MPEG-4 video transmission over IEEE 802.11e EDCA and IEEE 802.11n in heavy load. The simulation parameters and foreman QCIF video characteristics have shown in table 1 and 2 respectively. The IEEE $802.11 \mathrm{n}$ simulation parameters are shown in table 3, Simulation results show that Comparison of average PSNR, throughput and frame losses is shown in Table 4.

Table 1: Simulation parameter

\begin{tabular}{|c|c|c|c|c|}
\hline & VoIP & Video & $\begin{array}{l}\text { Back- } \\
\text { ground }\end{array}$ & $\begin{array}{l}\text { Best } \\
\text { Effort }\end{array}$ \\
\hline $\begin{array}{l}\text { Transport } \\
\text { protocol }\end{array}$ & UDP & UDP & UDP & TCP \\
\hline $\begin{array}{l}\text { Access } \\
\text { Category }\end{array}$ & $\mathrm{AC0}$ & $\mathrm{AC} 1$ & AC2 & AC3 \\
\hline
\end{tabular}




\begin{tabular}{|l|l|l|l|l|}
\hline Packet size & $1500 \mathrm{~B}$ & $1500 \mathrm{~B}$ & $1500 \mathrm{~B}$ & $1500 \mathrm{~B}$ \\
& & & & \\
\hline Sending rate & $64 \mathrm{Kbps}$ & $512 \mathrm{Kbps}$ & $256 \mathrm{Kbps}$ & $256 \mathrm{Kbps}$ \\
& & & & \\
\hline
\end{tabular}

Table 2: Total video packets and frames in video source (foreman)

\begin{tabular}{|c|c|c|c|c|c|}
\hline & \multicolumn{4}{|c|}{ Number of Packets } & Total \\
\hline Video & Format & $\mathrm{I}$ & $\mathrm{P}$ & B & \\
\hline Foreman & QCIF & 237 & 149 & 273 & 659 \\
\hline
\end{tabular}

\begin{tabular}{|l|l|l|l|l|l|}
\hline & \multicolumn{3}{|l|}{ Number of Frames } & $\begin{array}{l}\text { Total } \\
\text { Frames }\end{array}$ \\
\hline Video & Format & I & P & B & \\
\hline Foreman & QCIF & 45 & 89 & 266 & 400 \\
& & & & & \\
\hline
\end{tabular}

Table 3: IEEE 802.11n simulation parameter

\begin{tabular}{|l|l|}
\hline Parameter & Value \\
\hline CBR Interval time & $80 \mu \mathrm{sec}$ \\
\hline Packet Size & 1500 bytes \\
\hline Block Acknowledge type & 0 (none) \\
\hline RD-Reverse Direction & 0 \\
\hline Aggregation Size & 16383 bytes \\
\hline Flag for Contention Free Burst (cbr_) & 0 \\
\hline Number of Antenna & 4 \\
\hline MIMO System & 1 \\
\hline
\end{tabular}

This simulation compare performance of foreman video in high load traffic (VoIP 1 sources, Video 3 sources, CBR 1 source and FTP 1 source) for traditional AODV and purposed EAODV routing algorithm in in this simulation on IEEE 802.11n. The average PSNR, throughput, end-to-end delay were checked for both routing algorithms.
Table 4: Comparison of average PSNR, throughput and frame losses

\begin{tabular}{|l|l|l|l|l|l|l|}
\hline Routing & Average & \multirow{2}{*}{$\begin{array}{l}\text { Throughp } \\
\text { Algorithm }\end{array}$} & $\begin{array}{l}\text { PSNR } \\
\text { ut (Kbps) }\end{array}$ & number of Frame Losses \\
\cline { 4 - 8 } & & & I & P & B & Total \\
\hline ADOV & 33.254148 & 641.15 & 1 & 7 & 38 & 46 \\
\hline EAODV & 34.454985 & 677.62 & 0 & 1 & 77 & 78 \\
\hline
\end{tabular}

The comparison of average PSNR, throughput and frame losses show in table 4. The purposed algorithm EAODV give better average PSNR, throughput and less I frame (the main key frame in video which neither is not regenerated at destination) losses. Similarly comparison of PSNR and endto-end delay shown in figure 2 and 3 respectively. The purposed algorithms have also less end-to-end delay.

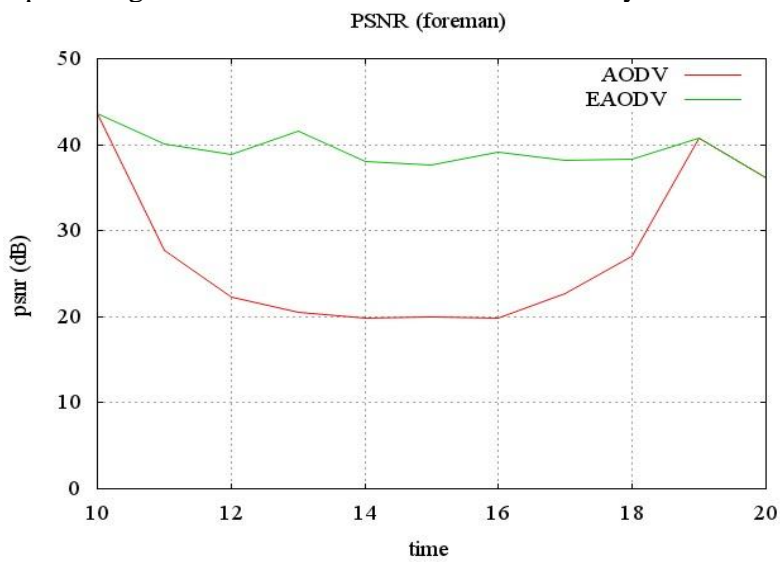

Figure 1: Comparison of PSNR

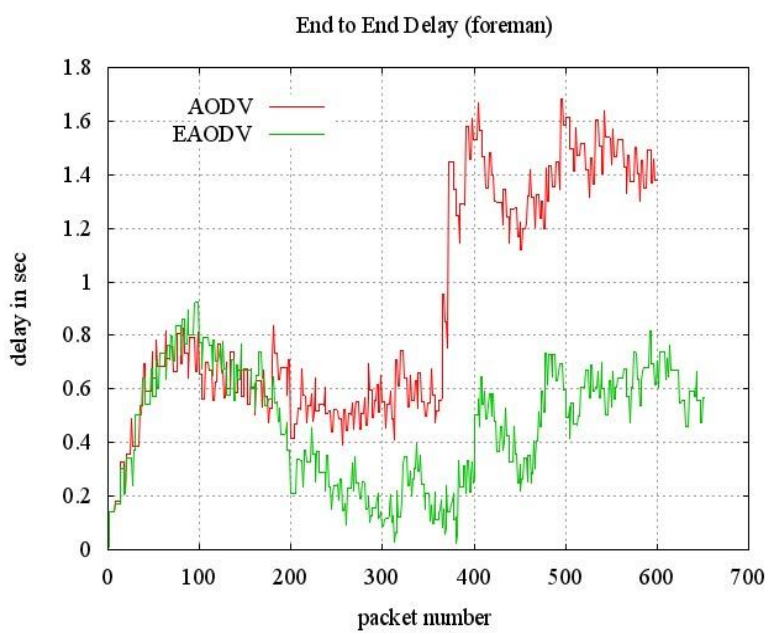

Figure 2: Comparison of End-to-End Delay

Three YUV video are played: original foreman_qcif.yuv, foreman_qcif_aodv.yuv and foreman_qcif_eaodv.yuv in yuvviewer.exe utility [30,31]. The comparison of received videos are shown below in figure. 3 

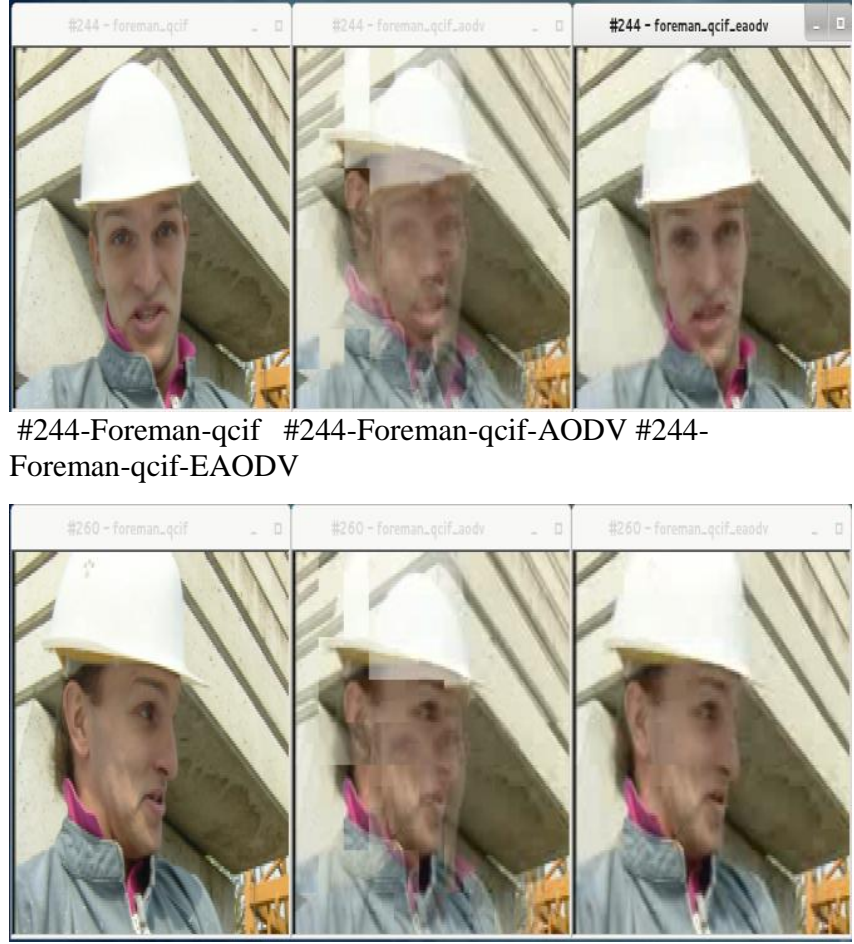

\#260-Foreman-qcif \#260-Foreman-qcif-AODV \#260-

Foreman-qcif-EAODV

(a) Comparison between frame 244 to 260

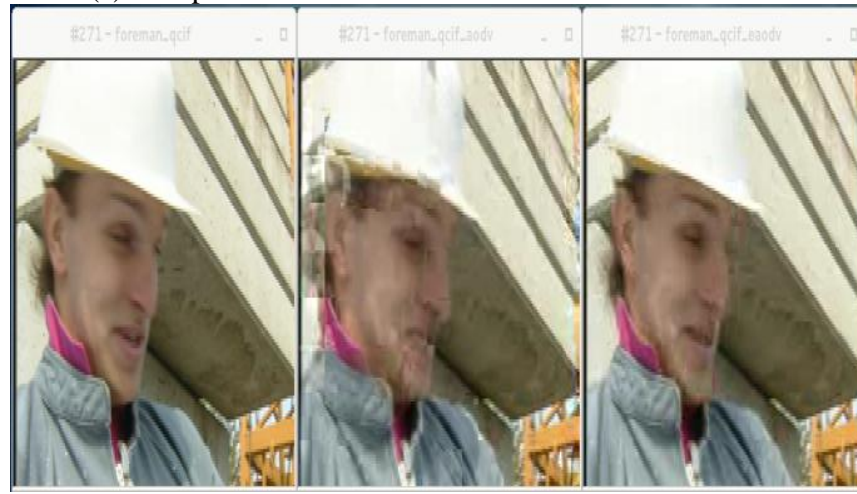

\#271-Foreman-qcif \#271-Foreman-qcif-AODV \#271-

Foreman-qcif-EAODV

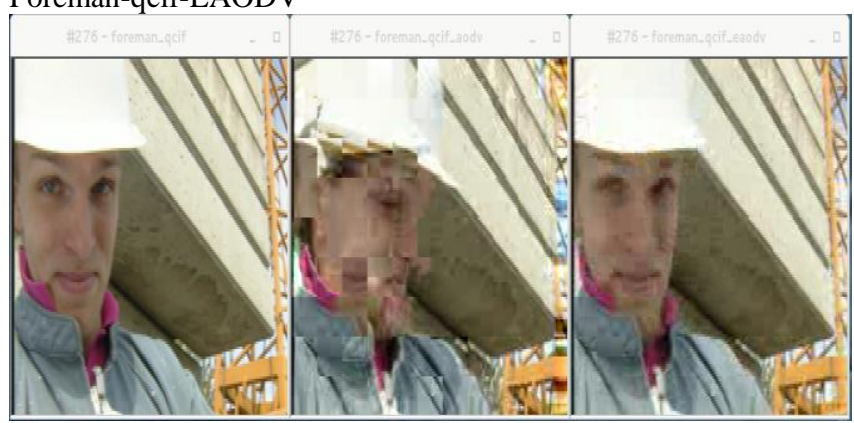

\#276-Foreman-qcif \#276-Foreman-qcif-AODV \#276-

Foreman-qcif-EAODV

(b) Comparison between frame 271 to 276

Figure 3: Comparison of received yuv using AODV and EAODV

The foreman QCIF video has total 400 frames. The figure 3 shows the comparisons of received yuv video. In figure 3(a) frame from 244 to 260 the EAODV give better output than AODV. Similarly in figure 3(b) frame from 271 to 276
EAODV give better output than AODV. Other than above frames rest of the frames received in good quality.

\section{CONCLUSION}

The purposed algorithm is simulated over IEEE 802.11n standard simulation parameter in heavy load environment with background traffic. QoS performance parameters i.e. PSNR, average PSNR, throughput, frame losses and end-toend delay are measured and compared for both the proposed and traditional algorithms. Proposed EAODV routing algorithm gave better result than the traditional AODV routing algorithm. Received video was also compared for both the algorithms, the EAODV give better output and received more good quality frames than AODV. These performance metrics are used to know how the characteristics of the EAODV protocol in CDNs differs in different parameters scenarios. This work consists of conditional link study and performance phase for the provision of quality of service metrics in CDN for large scale networks.

\section{REFERENCES}

[1] D. Doval and D. O'Mahony, "Overlay Networks: A Scalable Alternative for P2P”. IEEE Internet Computing, Vol. 7, No. 4, pp79-82, 2003.

[2] N. M. Mosharaf Kabir Chowdhury and Raouf Boutaba, "Network Virtualization: State of the Art and Research Challenges", IEEE Communications Magazine, pages 20-26, July 2009.

[3] S. Iyer, A. Rowstron, and P. Druschel, "Squirrel: A decentralized peer-to-peer web cache," in Proceedings of the 21st Symposium on Principles of Distributed Computing (PODC), Monterey, California,USA, July 21 242002

[4] J. Jannotti, D. K. Gifford, K. L. Johnson, M. F. Kaashoek and J. O'Toole, "Overcast: Reliable Multicasting with an Overlay Network", Proceedings of the 4th Symposium on Operating Systems Design and Implementation, pp. 197-212, 2000.

[5] Lakshminarayanan Subramanian, Ion Stoica, Hari Balakrishnan, Randy H. Katz, "OverQos: an overlay based architecture for enhancing internet QoS" Proceedings of the 1st conference on Symposium on Networked Systems Design and Implementation, pages: 6-6, 2004

[6] F. Dabek, J. Li, E. Sit, J. Robertson, M. F. Kaashoek, and R. Morris. Designing a DHT for low latency and high throughput. In USENIX First Symposium on Nerworked Systems Design and Implementation(NSDI'04), Mar. 2004.

[7] C. Plaxton, R. Rajaraman, and A. Richa, "Accessing nearby copies of replicated objects in a distributed environment," in Proceedings of the 9th Annual ACM Symposium on Parallel lgorithms and Architectures, 1997.

[8] “Akamai technologies." http://www.akamai.com/, June 2005.

[9] Turner, J., andWaldvogel, M., "Dimensioning server access bandwidth and multicast routing in overlay network", in Proceedings of NOSSDAV, Jun 2001.

[10] Banerjee, S., Kommareddy, C., Kar, K., Bhattacharjee S., and Khuller, S., "Construction of an efficient overlay 
multicast infrastructure for real-time applications," in Proceedings of IEEE INFOCOM, April 2003.

[11] "Gnutella protocol specification." http://www9.limewire.com/developer/ gnutella protocol 0.4.pdf, May 2002.

[12] Stoica, I., Morris, R., Karger, D., Kaashoek, F., and alakrishnan, H. B., "Chord: A peer-to-peer lookup service for Internet applications," in Proceedings of ACM SIGCOMM, (San Diego, CA), September 2001.

[13] Castro, M., Druschel, P., Kermarrec, A., Nandi, A Rowstron, "Split stream: High-bandwidth multicast in cooperative environments," in Proceedings of 19th ACM Symposium on Operating Systems Oct 2003.

[14] "Kazaa.” http://www.kazaa.com, May 2002.

[15] "PlanetLab." http://www.planet-lab.org/, May 2004.

[16] G. Peng, “CDN: Content Distribution Network", Technical Report TR-125, Experimental Computer Systems Lab, Department of Computer Science, State University of New York, Stony Brook, NY 2003

[17] J. Lu, "Signal processing for Internet video streaming: A review," in linage and Video Processing 2000, , Proceedings of SPIE, Vol. 3974, pp. 246-259. January 2000.

[18] S. Deering, "Host Extension for IP Multicasting", RFC1112. August, 1989.

[19] B. Quinn and K. Almeroth, "IP Multicast Applications: Challenges and Solutions", Internet Engineering Task Force (IETF) Internet Draft. March 2001.

[20] John G. Apostolopoulos, Wai-tian Tan, Susie J Wee, "PERFORMANCE OF A MULTIPLE DESCRIPTION STREAMING MEDIA CONTENT DELIVERY NETWORK", IEEE: 189-192, 2002.

[21] Jussara M. Almeidaa, Derek L. Eagerb, Michael Ferrisa, Mary K. Vernona "Provisioning Content Distribution Networks for Streaming Media” IEEE: 1746-1755, 2002.
[22] E. M. Royer and C. Toh, "A review of current routing protocols for Ad hoc mobile wireless networks," IEEE personal communication, pp 46-55, Apr 1999.

[23] C.E. Perkins and E.M. Royer, "Ad hoc on demand Distance Vector routing mobile computing systems and applications", Proceedings WMCSA, Second IEEE Workshop, pp 90-100, 1999.

[24] D.B. Johnson and D.A Maltz, "Dynamic source routing in adhoc wireless networks", T. Imielinski, H.Korth (Eds.), Mobile Computing, Kluwer Academic Publishers, pp.153-181, 1996.

[25] Vincent D. park and M. scott corson, "Temporally ordered Routing Algorithm (TORA) version 1: Functional specification", Internet draft, draft - ieftmanet- tora - spec-01.txt, August 1998.

[26] C.K. Toh, "Associativity Based Routing For Ad Hoc Mobile Networks", Wireless Personal Communications Journal, Special Issue on Mobile Networking and Computing Systems, pp 103-139, March 1997.

[27] R. Dube et al., "Signal stability based adaptive routing for ad hoc mobile networks", IEEE Pers. Comm., pp 3645, Feb 1997.

[28] Y.B. Ko and N.H. Vaidya. "Location Aid Routing (LAR) in mobile ad hoc networks", In Proc. ACM/IEEE MOBICOM, Oct. 1998.

[29] Annapurna Singh and Shailendra Mishra, "Performance Analysis of Reactive Routing Protocols in Mobile Ad hoc Networks," International Journal of Computer Science and Network Security, Vol.10, No. 8, pp. 141145, Aug 2010.

[30] http://hpds.ee.ncku.edu.tw/ jhlin5/ns2/crosslayer/NS2_crosslayer.htm,09 March 2013

[31] http://csie.nqu.edu.tw/smallko/ns2/myEvalvid.htm,09 March 2013 